\title{
An Effective Anticancer Nano-approach for Melanoma and Breast Cancers Using Annona muricate Gold Nanoparticles
}

\author{
Muhammad Imran ${ }^{1}$, Ghaleb Husseini ${ }^{2}$, Nahid Awad ${ }^{2}$, Vinod Paul ${ }^{2}$, \\ Babiker M El-Haj ${ }^{3}$ and Heyam Saad $\mathrm{Ali}^{4 *}$ \\ ${ }^{1}$ International Center for Chemical and Biological Sciences, H.E.J. Research Institute \\ of Chemistry, University of Karachi, Pakistan \\ ${ }^{2}$ Department of Chemical Engineering American University of Sharjah, Sharjah, \\ $U A E$ \\ ${ }^{3}$ Department of Pharmaceutical Sciences, College of Pharmacy and Health Sciences, \\ University of Science and Technology of Fujairah, Fujairah, UAE \\ ${ }^{4}$ Pharmaceutics Department, Pharmacy College, University of Khartoum, \\ Khartoum, Sudan \\ *Corresponding Author: Heyam Saad Ali, Pharmaceutics Department, Pharmacy \\ College, University of Khartoum, Khartoum, Sudan.
}

\author{
Received: August 08, 2021 \\ Published: August 18, 2021 \\ (C) All rights are reserved by Heyam Saad Ali., \\ et al.
}

\begin{abstract}
Chemotherapy is preferred for the treatment and management of cancer; however, its efficacy is hindered by the off-target side effects of the currently used synthetic drugs. Thus, anticancer drugs with higher safety and efficacy from renewable resources are needed. Nanostructured particles can enhance the drug specificity for target tissues, thus enhancing their clinical efficacy and safety. The synthesis of Annona muricata gold nanoparticles is reported for enhancing its anti-cancer potential. Green nanoparticles were synthesized through reduction of gold with Annona muricata followed by their extensive characterization through atomic force microscope, UV-spectrophotometer, zetasizer, and FT-R. They were investigated for their anticancer activity against two melanoma and one breast cancer cell line. The nanoparticles were rounded in shape and were monodispersed with $89.34 \pm 2.76 \mathrm{~nm}$ size and -22.41 \pm 0.27 zeta potential. FT-IR study showed the hydroxyl and carbonyl groups of Annona muricata were involved in the stabilization of nanoparticles. The extract anticancer activity significantly improved against all cancer cells upon loading on the surfaces of the synthesized NPs. The findings suggest that Annona muricata gold nanoparticles can lead to promising therapeutic anticancer results, however, to reveal their anticancer effectiveness at molecular levels, further in vivo studies are required.
\end{abstract}

Keywords: Annona muricata; Gold Nanoparticles; Synthesis; Anticancer Activity

\section{Introduction}

Cancer has been a major killer for a long time, which brings a huge terror to human beings globally [1]. It is the world's second most prevalent disease with the highest mortality, after cardiac problems [2-4]. In the United Arab Emirates (UAE), cancer is the third most common cause of death after cardiac disease and accidents [5]. The UAE Ministry of Health has reported approximately 500 deaths annually, while the world health organization reported increasing to 2079 in 2018 [6]. Millions of dirhams are currently being invested in the UAE to cure advanced cases of cancer, but the 
disease still remains at an alarming state. Among the popular cancers in the UAE, breast cancer is the main form of malignancy identified in the population of the UAE [7,8]. Similarly, one of the most common male malignancies in the UAE is skin cancer, with a 14.5 percent prevalence rate $[9,10]$.

A massive issue restricting the effectiveness of most commonly used chemotherapeutic drugs is their non-selectivity or non-specificity to tumor tissues and cells [11]. In healthy tissues and organs, this has contributed to the major side effects of these medications [12]. This non-selectivity or non-specificity against tumor tissues and cells produces toxicity that impedes successful chemotherapy [13]. Besides, the emergence of cancer cells resistant to the chemotherapy agent has posed a new challenge, resulting in unsatisfactory clinical results [14]. Thus, there is an immediate need to explore renewable resources for alternative anticancer drugs that demonstrate enhanced therapeutic efficacy with fewer side effects [15].

Plants provide animals and humans with an ongoing source of medicine; they have been used in rudimentary forms such as liniments, decoctions, infusions, ointments, syrups, and powders since ancient times. In modern times, herbal medicines are used by both advanced and developing countries to enhance their health $[16,17]$. Over the last three decades, natural products have gained considerable medicinal significance as innovative, secure, efficient, and affordable therapeutic agents [18-21]. Likewise, in numerous scientific articles, the potential of plant-derived compounds to inhibit different levels of tumor growth and related inflammatory processes has been documented [22]. These studies demonstrate the efficacy of natural products in the cure and control of cancer [23-25]. Although 50 percent of modern pharmaceutical medications are produced from plants in clinical practice, many of them have significant anti-cancer effects [26]. Owing to their richness in diverse phytoconstituents, versatile chemical composition, improved therapeutic efficacy, and safety, medicinal plants need to be further explored for their chemotherapeutic effects [26-29].

Annona muricata (A. muricata) is a species of the Annonaceae family that has been extensively studied over the past years because of its medicinal properties. The plant has been extensively reported for its anticancer activities. A. muricata leaves have been shown to possess anti-carcinogenic effects against colonic aberrant crypt foci induced by azoxymethane in rats $[30,31]$. An extract of ethanol from Muricata leaves has exhibited enhanced antitumor efficacy in murine models compared to curcumin [32]. In another research, an aqueous extract of commercial powder capsules comprising A. muricata leaf and stem demonstrated anti-tumor and anti-metastatic function against pancreatic tumors in murine models [33,34]. Various other studies have also reported the anticancer effects of the plant or its isolated bioactive compounds and have documented its different anticancer mechanisms [35].

As an interesting innovation for the production of anticancer drug nanoparticles, nanotechnology has been introduced. Gold nanoparticles (AuNPs) have an outstanding controlled size, stability, and biocompatibility characteristics as the best drug nanocarriers, enabling them effective agents in the diagnosis and cure of cancer [36-38]. The production of nanoparticles by using a process of chemical reduction leads to the production of toxic compounds adsorbed to the surface of the particles, thus prevent their use for biomedical applications $[38,39,41]$. Therefore, researchers are currently employing different biological approaches to concentrate on the production of biocompatible and eco-friendly nanoparticles. Green synthesis of nanoparticles using plant extract has been highly fascinating due to their higher safety, ease of synthesis, and stability [42-44]. Furthermore, the synthesis of nanoparticles with extracts of medicinal plants enhance their pharmacological efficacy due to their smaller nano-size, increased interaction biological systems as well as their accumulation in target sites [45-47].

\section{Purpose of the Study}

The purpose of this research study is the synthesis of $A$. muricata leaves ethanolic extract stabilized AuNPs and their evaluation for anticancer activity against melanoma and breast cancer.

\section{Experimental Methodology \\ Collection of plant material and identification}

Fresh leaves of $A$. muricata were purchased from the Abu Dubai market, UAE, during August 2021. The plant was identified by Dr. Ibrahim Futuh. The voucher of the specimen was deposited in the herbarium of the Department of Pharmaceutical Chemistry and Natural Products, Pharmacy, University of science and technology of Al Fujairah, Al Fujairah, UAE.

\section{Ethanolic extract preparation}

After collection, the leaves of the plant were thoroughly rinsed and dried for 10 days at room temperature. The shade dried sam- 
ples were then ground into a coarse powder with an electric mixer and placed in a polythene bag at room temperature. The content was then immersed in ethanol (80 percent) for 15 days. Then the extract was filtered using Whatman's No; 1 filter paper. The extract was concentrated to dryness under lower power at temperature $\left(45^{\circ} \mathrm{C}\right)$ using a rotary evaporator.

\section{Synthesis of gold nanoparticles (AM-AuNPs)}

The synthesis of the ethanol extract $A$. muricata AuNPs (AMAuNPs) was initially configured by combining various concentrations $(0.5-2.5 \mathrm{mg} / \mathrm{mL}$ ) of ethanol extract $A$. muricata with gold solution $1 \mathrm{~mm}$ ) at $1: 1 \mathrm{v} / \mathrm{v}$ ratio. At the ambient temperature, the mixture was stirred at $100 \mathrm{rpm}$ for $6 \mathrm{~h}$. Initially, the color alters to deep purple from light yellow, suggesting the synthesis of AMAuNPs. The NPs were further characterized by the UV-visible spectrophotometer (UV-240, Shimadzu, Kyoto, Japan) for their distinctive surface plasmon resonance (RSP) peak. For the effects of different concentrations (0.2 - $1 \mathrm{mM})$ of the gold solution, the NPs were further investigated.

Characterization surface morphology, size, zeta potential, and PDI

An atomic force microscope (AFM, Agilent 5500) was employed to examine the surface morphology of the synthesized NPs. AMAuNPs were placed in a dust-free compartment at room temperature on a mica slide and air dried. The slide was positioned on the microscope, viewed in non-contact mode, and the images were captured. Zetasizer (ZS-90 Malvern instruments, the UK) was used to evaluate the scale, PDI, and zeta potential. Appropriately diluted samples were collected in a disposable plastic cuvette for size and PDI determination, and measurements were taken at $25^{\circ} \mathrm{C}$. Diluted samples were steadily injected into capillary cells for zeta potential evaluation and observed at $25^{\circ} \mathrm{C}$. Measurements with water as a dispersant were taken in triplicate.

\section{FT-IR analysis}

For the purpose to examine the participation of the functional groups of ethanolic extract of $A$. muricata in the reduction/ stabilization of AM-AuNPs, FT-IR analysis was carried out. KBr powder was combined with dried samples of ethanolic extract and AMAuNPs and pressed for self-supporting disks. The IR spectra were obtained by an IR spectrometer (Shimadzu, Kyoto, Japan) in the rage os 900 to $4000 \mathrm{~cm}^{-1}$.

\section{Anticancer activity}

MCF-7, breast cancer cells, was gifted from Prof Maiweh Hamed (University of Sharjah, UAE). Metastatic melanoma [MM-138] and primary melanoma FM-55, melanoma cells were purchased from Sigma Aldrich, UK from ECACC cell lines collection. In DMEM, the MCF-7 cells were cultured, whereas the melanoma cell lines were preserved in RPMI-1640 medium with glutamine and HEPES supplementation. The media contained antibiotics (50 units/mL streptomycin and 50 units/mL penicillin) and FBS (10\%). The cells were incubated in a humified aseptic containing 5 percent $\mathrm{CO}_{2}$ at $37^{\circ} \mathrm{C}$ environment. MTT assays were used to examine the cytotoxicity of ethanolic extract of $A$. muricata, and AM-AuNPs at varying concentrations $(62.5,125,250$, and $500 \mu \mathrm{g} / \mathrm{mL})$ and negative control against these cells. The cells culturing was carried out in 96well plates at a concentration of $8.0 \times 103$ cells/well in a culture medium $(200 \mu \mathrm{L})$. The initial medium was replaced after incubation for 24 hours and a new medium $(200 \mu \mathrm{L})$ containing different concentrations ( $62.5-500 \mu \mathrm{g} / \mathrm{mL}$ ) of test samples was introduced. Without the test sample, cells were seeded with negative regulation media. Cell cultivation was then maintained for 24h. MTT (20 $\mu \mathrm{L} ; 5 \mathrm{mg} / \mathrm{mL}$ ) solution in PBS was applied to each well. The medium containing the unreacted dye was extracted after incubating the cells for $4 \mathrm{~h}$. The purple formazan crystals collected were immersed in $200 \mu \mathrm{L}$ per well of dimethyl sulphoxide (DMSO) and the absorption was assessed at $570 \mathrm{~nm}$ wavelength in microplate readers (ELx808, BioTek, USA). The toxicity of the cells in the test samples was measured adequately.

\section{Statistical analysis}

The data were represented as mean \pm SEM. All performed tests were conducted at triplicate. To draw the graphs, the GraphPad Prism was employed. For comparison, the two-way ANOVA accompanied by the Bonferroni test was used. P values $<0.05$ were considered to be statistically significant. $P$ values less than or equal to 0.05 were assumed to be statistically significant.

\section{Results and Discussion}

The AuNP synthesis is commonly achieved via the reduction of the gold atoms from a charged state to a normal state. In this research, the ethanol extract of $A$. muricata has been used both as a reducing and as a stabilizing agent. Plant-based extracts have been used for AuNP green synthesis. Such products reduce the use of 
dangerous and harmful synthetic reducing agents such as sodium dimethylformamide, hydrazine hydrate, and borohydride [25]. The characteristic absorption of the extract solution is shown in figure 1A. Maximum absorbance was detected for $2.5 \mathrm{mg} / \mathrm{mL}$ of the extract solution when various concentrations $(0.5-2.5 \mathrm{mg} / \mathrm{mL})$ of A. muricata ethanol extract were blended with $1 \mathrm{mM}$ gold solution in a $1: 1 \mathrm{v} / \mathrm{v}$ ratio. It showed a peak of Surface Plasmon Resonance (SPR) at $538 \mathrm{~nm}$ with 1.45 absorbance, indicating the peak synthesis of AM-AuNPs as depicted in figure 1B. The intensity of the characteristic SPR peak dropped with a concurrent reduction of the concentration of $A$. muricata extract. 12 As the concentration of A. muricata ethanolic extract reduced, the intensity of the characteristic SPR peak dropped significantly. This indicates that for adequate reduction and stabilization of NPs, lower concentrations of A. muricata ethanolic extract are not enough. Likewise, keeping the A. muricata ethanolic extract concentration at $2.5 \mathrm{mg} / \mathrm{mL}$, the effects of gold concentration were also examined. The results indicated maximum absorbance for the highest gold concentration used, i.e. $1 \mathrm{mM}$. A distinctive SPR peak with maximum absorbance was displayed, confirming the peak synthesis of NPs as seen in figure $1 c$. The reducing concentration of gold triggered the intensity of the characteristic SPR to decrease. Therefore, the ideal parameters for the synthesis of AM-AuNPs with the enhanced intensity of the characteristic SPR peak were estimated to be $2.5 \mathrm{mg} / \mathrm{mL}$ of $A$. muricata ethanolic extract and $1 \mathrm{mM}$ of gold mixed in a ratio of $1: 1 \mathrm{v} / \mathrm{v}$.
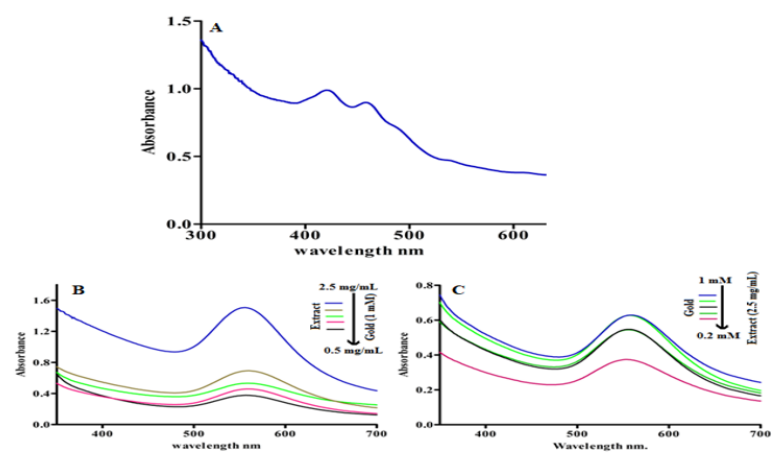

Figure 1: (A) UV-visible spectrum A. muricata ethanolic extract, (B) and (C) UV-visible spectra showing effects of the concentrations of $A$. muricata ethanolic extract and gold on the synthesis of AM-AuNPs.
Characterization of AM-AuNPs

Surface morphology, size, PDI, and zeta potential

Surface morphology, zeta potential, PDI, and size of AM-AuNPs were explored by zetasizer and AFM respectively. AM-AuNPs showed smooth spherical surface morphologies as indicated in figure 2. As shown in figure $3 \mathrm{~A}$. AM-AuNPs had a mean surface charge of $-22.41 \pm 0.27$. AM-AuNPs indicated a mean particle size of 89.34 $\pm 2.76 \mathrm{~nm}$ as shown in figure 3B. The relatively greater size of AMAuNPs may be due to A. Muricata ethanol extract that induces their reduction/stabilization. The NPs showed a PDI value of $0.19 \pm 0.01$, indicating that they are highly monodispersed as indicated in figure 3B. The size of NPs on the nano range scale influences the drug delivery systems' in-vivo performance as well as physical stability. When analyzed by zetasizer, AuNPs were observed to be good in nano-size. In addition, this size analysis validates spectrophotometric screening where NPs have been identified with a narrow and sharp SPR peak. In addition, nano-range particles show reduced toxicity in vivo relative to their larger counterparts [48-50]. Nano range size was shown by the synthesized NPs, predicting their better invivo efficiency and physical stability. Another significant aspect of a nano-based drug delivery system is the Zeta potential, as it maximizes the physical stability of the formulations by avoiding particle aggregation and fusion. The greater zeta potential also guarantees interactions of biological membranes with drug delivery systems and, therefore, leads to the greater therapeutic effectiveness of the loaded drugs [51,52].

The A. muricata ethanolic extract IR spectrum displays characteristic peaks of absorption for-OH groups at $3365 \mathrm{~cm}^{-1}$ and $\mathrm{NH}-$

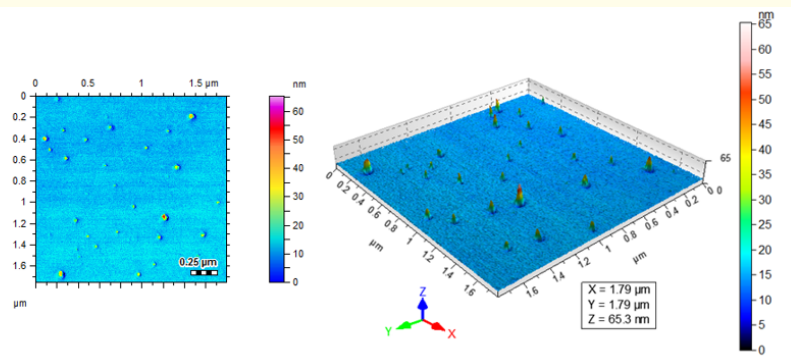

Figure 2: Surface morphological analysis of the synthesized AM-auNPs. 

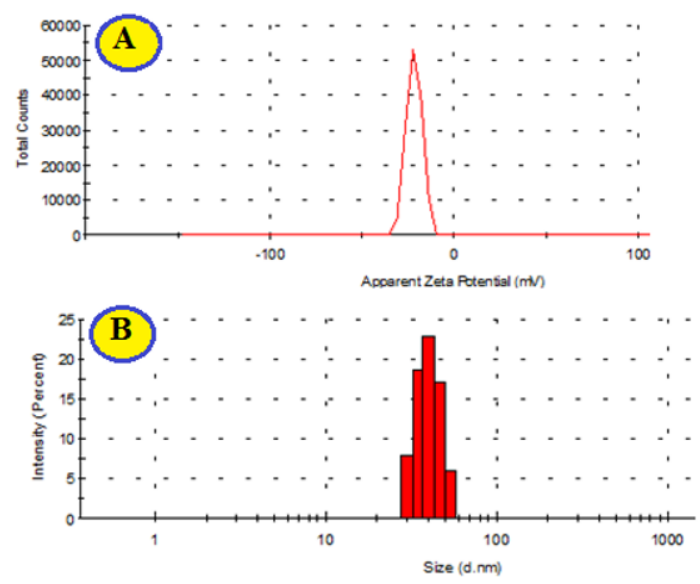

Figure 3: 10 (A) zeta potential and (B) the average size of the synthesized AM-AuNPs.

$\mathrm{C}=0$ functional groups at $1793 \mathrm{~cm}^{-1}$. Likewise, it shows characteristic peaks for $\mathrm{C}=\mathrm{O}, \mathrm{C}=\mathrm{C}$ (aromatic), and $\mathrm{C}-\mathrm{O}-\mathrm{C}$ functional groups at 1640,1529 , and $1040 \mathrm{~cm}^{-1}$ as shown in figure 4 , respectively. At $3341 \mathrm{~cm}^{-1}$ for-OH groups, $1715 \mathrm{~cm}-1$ for $\mathrm{NH}-\mathrm{C}=0$ functional groups, and $1608 \mathrm{~cm}^{-1}$ for $\mathrm{C}=0$ functional groups, the IR spectra of AM-AuNPs reveal their characteristic peaks. Similarly, for functional groups $\mathrm{C}=\mathrm{C}$ (aromatic) and $\mathrm{C}-\mathrm{O}-\mathrm{C}$, it also displays characteristic absorption peaks at 1528 and $1020 \mathrm{~cm}^{-1}$ respectively. The transition in absorption peaks from $3365.2 \mathrm{~cm}^{-1}$ to $3379 \mathrm{~cm}^{-1}$ and from $1040 \mathrm{~cm}-1$ to $1020 \mathrm{~cm}^{-1}$ indicates that $A$. muricata ethanolic extract functional groups-OH and $\mathrm{C}-\mathrm{O}-\mathrm{C}$ are actively engaged in the AMAuNP degradation and stabilization.

Anticancer activity The MTT cytotoxicity assay is widely used in vitro to determine the viability of cancer cell lines. The in vitro cytotoxicity of anticancer drugs is normally assessed by this technique [28]. In the current research, the synthesized AM-AuNPs and plant ethanolic extract were screened for their anticancer activity against two melanoma (Metastatic melanoma [MM-138] and primary melanoma [FM-55]) and one breast cancer cell lines using MTT assay. When tested against melanoma cells MM-138, the synthesized AM-AuNPs revealed improved anticancer activity as compared to the simple ethanolic extract. At the highest concentration of $500 \mu \mathrm{g} / \mathrm{mL}$, AM-AuNPs showed $78.98 \pm 2.52 \%$ cytotoxicity as

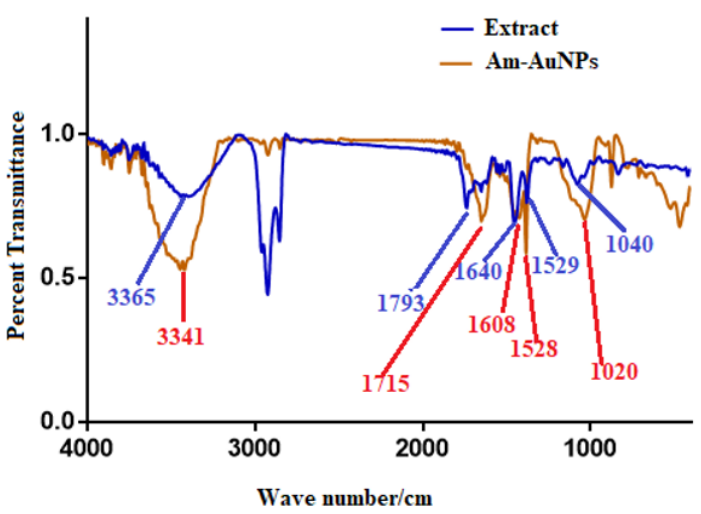

Figure 4: FT-IR analysis of plant ethanolic extract and its stabilized NPs.

compared to $61.07 \pm 2.87 \%$ cytotoxicity shown by the simple extract of the plant (Figure 5A). Similarly, the synthesized AM-AuNPs showed higher cytotoxicity against the melanoma FM-55 cells as compared to the simple extract of the plant. At the highest concentration of $500 \mu \mathrm{g} / \mathrm{mL}$, AM-AuNPs showed $81.33 \pm 4.81 \%$ cytotoxicity as compared to $60.00 \pm 2.44 \%$ cytotoxicity shown by the simple extract of the plant (Figure 5B). In MCF-7 breast cancer cells, the AM-AuNPs exhibited improved anticancer activity compared to the simple extract. 13 In the case of MCF-7 breast cancer cells, the AMAuNPs demonstrated improved anticancer activity as compared to the simple extract. At the highest concentration of $500 \mu \mathrm{g} / \mathrm{mL}, \mathrm{AM}$ AuNPs showed $87.19 \pm 3.63 \%$ cytotoxicity as compared to $71.45 \pm$ $1.87 \%$ cytotoxicity shown by the simple extract of the plant (Figure 6).

In this research, the synthesized AM-gold nanoparticles displayed substantial cytotoxicity activity towards two cancer cells of melanoma and one breast cancer cell in a dose-dependent manner. As the concentration of synthesized gold nanoparticles enhanced, the cancer cell lines' viability proportion decreased drastically. It clearly indicates the possible cytotoxic activity of the synthesized AM-gold nanoparticles against the three cancer cell lines. The function of anticancer activity of AuNPs may be recognized by the adsorbed active molecules present in A. muricata ethanolic extract as reported by previous researches documenting plant AuNPs for an- 
ticancer activity [53-55]. Another possible reason for the improvement in the anticancer activity of the A. muricata ethanolic extract upon its loading on AuNPs can be the greater interaction of the synthesized NPs towards the membrane of the biological systems (the three cell lines in our case) [56-58]. Similarly, the smaller nano-size of the synthesized NPs can also increase the biological activity of the therapeutic substances adsorbed on their surfaces [59-61].
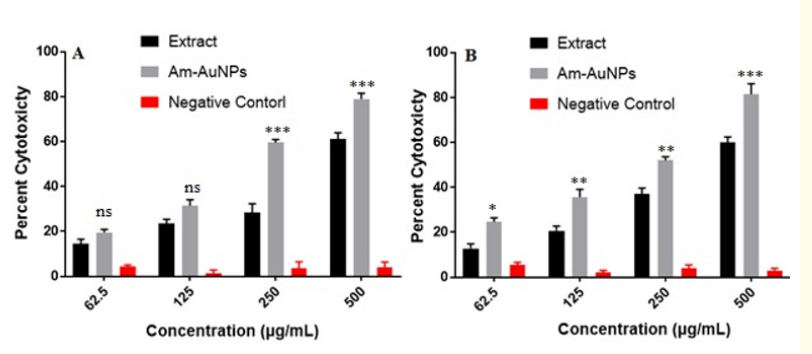

Figure 5: Anticancer activity of the synthesized NPs and the ethanolic extract against (A) MM-138 and (B) FM-55 melanoma cells.

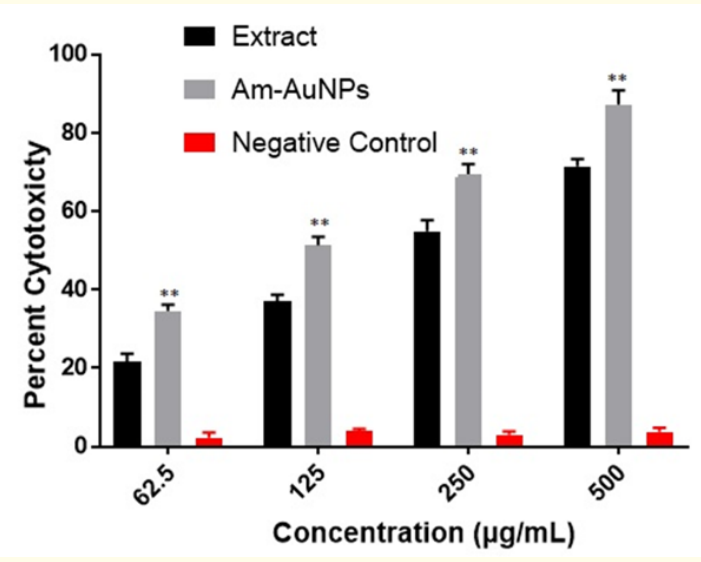

Figure 6: Anticancer activity of the synthesized NPs and the ethanolic extract against MCF-7 breast cancer cells.

\section{Conclusion}

In conclusion, we successfully synthesized gold nanoparticle from the A. muricata leaves extract and screened them for anti- cancer activity against melanoma and breast cancer cells. The synthesized NPs were highly monodispersed in the nanosized range and were spherical. Multi-functional groups of the extract were involved in the stabilization of the synthesized NPs. The extract anticancer activity significantly improved against all cancer cells upon loading on the surfaces of the synthesized NPs. However, further studies of the A. muricata gold nanoparticles may contribute to future anticancer therapies for breast and skin cancers.

\section{Acknowledgment}

This research was partly funded by a targeted grant from the University of Sharjah (grant no. 16010901011-P). The researcher would like to acknowledge Department of Chemical Engineering American University of Sharjah, Sharjah, UAE, for the support of the research project.

\section{Bibliography}

1. Bray F., et al. "Global cancer statistics 2018: GLOBOCAN estimates of incidence and mortality worldwide for 36 cancers in 185 countries". CA: A Cancer Journal for Clinicians 68 (2018): 394-424.

2. Bray F., et al. "Cancer Incidence in Five Continents: inclusion criteria, highlights from Volume $\mathrm{X}$ and the global status of cancer registration". International Journal of Cancer 137 (2015): 2060-2071.

3. Ginsburg 0., et al. “The global burden of women's cancers: a grand challenge in global health". Lancet 389 (2017): 847-860.

4. Torre L A., et al. "Global cancer incidence and mortality rates and trends - an update". Cancer Epidemiology, Biomarkers and Prevention 25 (2016): 16-27.

5. Loney T., et al. "An analysis of the health status of the United Arab Emirates: the 'Big 4' public health issues". Global Health Action 6 (2013): 20100.

6. Harbeck N., et al. "Breast cancer". Nature Reviews Disease Primers 5 (2019): 66.

7. Holt CP. "The scope of the UAE cancer problem". Emirates Medical Journal 3.3 (1985): 163-166.

8. Al-Shamsi H and S Alrawi. "Breast cancer screening in the United Arab Emirates: is it time to call for a screening at an earlier 
age?" Journal of Cancer Prevention and Current Research 9.3 (2018): 123-126.

9. Radwan H., et al. "The epidemiology of cancer in the United Arab Emirates: Systematic Review". Medicine 97.50 (2018).

10. Mahate A and Hamidi S. "Frontier efficiency of hospitals in United Arab Emirates: an application of data envelopment analysis". Journal of Hospital Administration 5.1 (2015): 7-17.

11. Boumahdi S and de Sauvage FJ. "The great escape: tumour cell plasticity in resistance to targeted therapy". Nature Reviews Drug Discovery 19.1 (2020): 39-56.

12. Galletti G., et al. "Mechanisms of resistance to systemic therapy in metastatic castration-resistant prostate cancer". Cancer Treatment Reviews 57 (2017): 16-27.

13. Harrington K., et al. "Optimizing oncolytic virotherapy in cancer treatment". Nature Reviews Drug Discovery 18.9 (2019): 689-706.

14. Riesco-Martinez M., et al. "Resistance to metronomic chemotherapy and ways to overcome it". Cancer Letter 400 (2017): 311-318.

15. Zhang H., et al. "Recombinant oncolytic adenovirus expressing a soluble PVR elicits long-term antitumor immune surveillance". Molecular Therapy - Oncolytics 20 (2020): 12-22.

16. Yeung KS., et al. "Herb-Drug Interactions in Cancer Care”. Oncology (Williston Park) 32.10 (2018): 516-520.

17. Singh D., et al. "Herbs-are they safe enough? An overview". Critical Reviews in Food Science and Nutrition 52.10 (2012): 876898.

18. Boon $\mathrm{H}$ and Wong J. "Botanical medicine and cancer: a review of the safety and efficacy". Expert Opinion on Pharmacotherapy 5.12 (2004): 2485-2501.

19. Werneke U., et al. "Potential health risks of complementary alternative medicines in cancer patients". British Journal of Cancer 90.2 (2004): 408-413.

20. Haefeli WE and Carls A. "Drug interactions with phytotherapeutics in oncology". Expert Opinion on Drug Metabolism and Toxicology 10.3 (2014): 359-377.
21. Solowey E., et al. "Evaluating medicinal plants for anticancer activity”. Scientific World Journal 2014 (2014): 721402.

22. Yao S., et al. "Development and evaluation of novel tumor-targeting paclitaxel-loaded nano-carriers for ovarian cancer treatment: in vitro and in vivo". Journal of Experimental and Clinical Cancer Research 37 (2018): 29.

23. Izzo AA and Ernst E. "Interactions between herbal medicines and prescribed drugs: a systematic review”. Drugs 61.15 (2001): 2163-2175.

24. SBM Ahmed., et al. "Studying the ShcD and ERK interaction under acute oxidative stress conditions in melanoma cells". The International Journal of Biochemistry and Cell Biology 112 (2019): 123-133.

25. Ovadje P., et al. "Advances in the research and development of natural health products as main stream cancer therapeutics". Evidence-based Complementary and Alternative Medicine (2015): 751348.

26. Wang Y., et al. "Antitumor effects of immunity-enhancing traditional Chinese medicine". Biomed Pharmacotherapy 121 (2020): :109570.

27. Omara T., et al. "Medicinal Plants Used in Traditional Management of Cancer in Uganda: A Review of Ethnobotanical Surveys, Phytochemistry, and Anticancer Studies". Evidencebased Complementary and Alternative Medicine 2020 (2020): 3529081-3529081.

28. BM El-Haj and S Ahmed. "Metabolic-Hydroxy and Carboxy Functionalization of Alkyl Moieties in Drug Molecules: Prediction of Structure Influence and Pharmacologic Activity". Molecules 25.8 (2020): 1937.

29. Yuan H., et al. "The Traditional Medicine and Modern Medicine from Natural Products". Molecules (Basel, Switzerland) 21.5 (2015): 559.

30. Zorofchian Moghadamtousi S., et al. "Annona muricata leaves induce $\mathrm{G}_{1}$ cell cycle arrest and apoptosis through mitochondria-mediated pathway in human HCT-116 and HT-29 colon cancer cells". Journal of Ethnopharmacology 156 (2014): :277289. 
31. Siti Mariam., et al. "Exploring the Leaves of Annona muricata L. as a Source of Potential Anti-inflammatory and Anticancer Agents". Frontiers in Pharmacology 9 (2018): :661.

32. Syed Najmuddin SU., et al. "Anti-cancer effect of Annona Muricata Linn Leaves Crude Extract (AMCE) on breast cancer cell line". BMC Complement Alternative Medicine 16.1 (2016): 311.

33. Joo D., et al. "Annona muricata L. extracts decrease melanogenesis in B16F10 mouse melanoma cells". Biomed Dermatology 1 (2017): 10.

34. George VC., et al. "Quantitative assessment of the relative antineoplastic potential of the n-butanolic leaf extract of Annona muricata Linn. in normal and immortalized human cell lines". Asian Pacific Journal of Cancer Prevention 13.2 (2012): 699704.

35. Yu-Min Koa., et al. "Annonacin induces cell cycle-dependent growth arrest and apoptosis in estrogen receptors- $\alpha$-related pathways in MCF-7 cells". Journal of Ethnopharmacology 137.3 (2011): : 1283-1290.

36. Wang B., et al. "Novel PEG-graft-PLA nanoparticles with the potential for encapsulation and controlled release of hydrophobic and hydrophilic medications in aqueous medium". International Journal of Nanomedicine 6 (2011): :1443-1451.

37. Wicki A., et al. "Nanomedicine in cancer therapy: challenges, opportunities, and clinical applications". Journal of Control Release 200 (2015): 138-157.

38. Marchetti C., et al. "Targeted drug delivery via folate receptors in recurrent ovarian cancer: a review". OncoTargets Therapy 7 (2014): 1223-1236.

39. Fang J., et al. "The EPR effect: unique features of tumor blood vessels for drug delivery, factors involved, and limitations and augmentation of the effect". Advanced Drug Delivery Reviews 63 (2011): 136-151.

40. Sun XL., et al. "Improved tumor uptake by optimizing liposome based RES blockade strategy". Theranostics 7 (2017): 319-28.

41. Imran Ali., et al. "Advances in nanoparticles as anticancer drug delivery vector: Need of this century". Current Pharmaceutical Design 26 (2020): 1637-1649.
42. BM El-Haj., et al. "Linking Aromatic Hydroxy Metabolic Functionalization of Drug Molecules to Structure and Pharmacologic Activity". Molecules 23.9 (2018): 2119.

43. Zhang X., et al. "Rabdosiarubescens Linn: green synthesis of gold nanoparticles and their anticancer effects against human lung cancer cells A549”. Artificial Cells 47.1 (2019): 2171-2178.

44. K Rao., et al. "Gum tragacanth stabilized green gold nanoparticles as cargos for Naringin loading: A morphological investigation through AFM". Carbohydrate Polymer 174 (2017): 243252.

45. Imran M., et al. "Glycoside-based niosomal nanocarrier for enhanced in-vivo performance of Cefixime". International Journal of Pharmaceutics 505.1-2 (2016): 122-132.

46. SBM Ahmed., et al. "A nuclear export signal and oxidative stress regulate ShcD subcellular localisation: A potential role for ShcD in the nucleus". Cellular Signalling 26.1 (2014): 32-40.

47. Sun B., et al. "Anticancer activity of green synthesised gold nanoparticles from Marsdeniatenacissima inhibits A549 cell proliferation through the apoptotic pathway". Artificial Cells 47.1 (2019): 4012-4019.

48. Sharma D., et al. "Biogenic synthesis of nanoparticles: A review”. Arabian Journal 8 (2019): 3576-3600.

49. Botteon CEA., et al. "Biosynthesis and characterization of gold nanoparticles using Brazilian red propolis and evaluation of its antimicrobial and anticancer activities". Scientific Report 11 (2021): 1974.

50. Sarvamangala D., et al. "Green synthesis of AgNP'S using Alternanthera Sessilis leaf extract [a natural source for ocular therapy]". International Journal of Innovative Science Engineering and Technology 3 (2014): 15000-15010.

51. Bhau BS., et al. "Green synthesis of gold nanoparticles from the leaf extract of Nepenthes khasiana and antimicrobial assay". Advanced Materials Letters 6 (2015): 55-58.

52. I Ali., et al. "Preliminary investigation of novel tetra-tailed macrocycle amphiphile based nano-vesicles for amphotericin B improved oral pharmacokinetics". Artificial Cells, Nanomedicine, and Biotechnology 46 (2017): S1204-S1214. 
53. Sharma D., et al. "Biogenic synthesis of nanoparticles: a review". Arabian Journal of Chemistry 12.8 (2019): 3576-3600.

54. Shukla R., et al. "Biocompatibility of gold nanoparticles and their endocytotic fate inside the cellular compartment: a microscopic overview". Langmuir 21 (2005): 10644-10654.

55. Dhas TS., et al. "Sargassum myriocystum mediated biosynthesis of gold nanoparticles". Spectrochimica Acta, Part A: Molecular and Biomolecular Spectroscopt 99 (2012): 97-101.

56. Heyam Saad Ali., et al. "Gold nanoparticles in cancer diagnosis and therapy". Book: Metal Nanoparticles for Drug Delivery and Diagnostic Applications 43-58.

57. Vijayan R., et al. "Indigofera tinctoria leaf extract mediated green synthesis of silver and gold nanoparticles and assessment of their anticancer, antimicrobial, antioxidant and catalytic properties". Artificial Cells 46 (2018): 861-871.

58. Ahmed SBM and Prigent SA. "Insights into the Shc family of adaptor proteins". 12 (2017): 1-17.

59. Fahradyan A., et al. "Updates on the Management of Non-Melanoma Skin Cancer (NMSC)”. Healthcare (Basel) 5 (2017): 82.

60. Chen JS., et al. "Inhibition mechanism of koji acid on polyphenol oxidase". Journal of Agricultural and Food Chemistry 58 (1991): 79-110.

61. Wang L., et al. "Green synthesis of gold nanoparticles from Scutellariabarbata and its anticancer activity in pancreatic cancer cell (PANC-1)". Artificial Cells 47.1 (2019): 1617-1627.

\section{Volume 5 Issue 9 September 2021}

(C) All rights are reserved by Heyam Saad Ali., et al. 